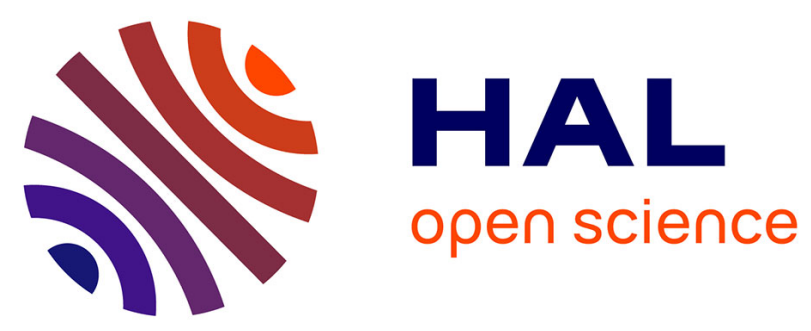

\title{
A Constrained Gauss-Newton Algorithm for Material Decomposition in Spectral Computed Tomography
}

\author{
Tom Hohweiller, Nicolas Ducros, Françoise Peyrin, Bruno Sixou
}

\section{To cite this version:}

Tom Hohweiller, Nicolas Ducros, Françoise Peyrin, Bruno Sixou. A Constrained Gauss-Newton Algorithm for Material Decomposition in Spectral Computed Tomography. ISBI 2018 IEEE International Symposium on Biomedical Imaging, IEEE, Apr 2018, Washington, United States. 10.1109/ISBI.2018.8363587 . hal-01825613

\section{HAL Id: hal-01825613 \\ https://hal.science/hal-01825613}

Submitted on 28 Jun 2018

HAL is a multi-disciplinary open access archive for the deposit and dissemination of scientific research documents, whether they are published or not. The documents may come from teaching and research institutions in France or abroad, or from public or private research centers.
L'archive ouverte pluridisciplinaire HAL, est destinée au dépôt et à la diffusion de documents scientifiques de niveau recherche, publiés ou non, émanant des établissements d'enseignement et de recherche français ou étrangers, des laboratoires publics ou privés. 


\title{
A Constrained Gauss-Newton Algorithm for Material Decomposition in Spectral Computed Tomography
}

\author{
Tom Hohweiller $^{\star} \quad$ Nicolas Ducros ${ }^{\star} \quad$ Françoise Peyrin ${ }^{\star \dagger} \quad$ Bruno Sixou $^{\star}$ \\ * Univ. Lyon, INSA-Lyon, Université Claude Bernard Lyon 1, UJM-Saint Etienne, CREATIS CNRS UMR 5220, Inserm U1206, F-69621, LYON, France \\ $\dagger$ The European Synchrotron Radiation Facility, Grenoble, France
}

\begin{abstract}
Spectral computed tomography exploits energy-resolved detectors to recover the material composition of an object. Material decomposition is a challenging nonlinear and ill-posed inverse problem. While regularization improves the decomposition, the resulting material maps do not satisfy physical constraints (e.g., positivity). In this work, we propose a fast second-order algorithm for constrained material decomposition. The proposed constrained Gauss-Newton algorithm is compared to a standard (unconstrained) Gauss-Newton algorithm on two realistic numerical phantoms. An improved decomposition is obtained for both phantoms. We also found that the constraints must be enforced progressively during the iterations.
\end{abstract}

Index Terms - Constrained minimization, spectral computed tomography, material decomposition, inverse problem

\section{INTRODUCTION}

Spectral computed tomography (CT) consists in acquiring energy-resolved projections that can be used to recover the concentration of the materials an object is made of. This modality can either exploit multiple X-ray source spectra or energy-resolved detectors. Of particular interest are the recent energy-resolved photon-counting detectors [1]. With such detectors, the numbers of photons within multiple energy bins are recorded considering only one X-ray source. Spectral CT is expected to have many applications in medical imaging, e.g., high-Z contrast agents imaging [2].

Spectral computed tomography assumes that the object can be decomposed onto some physical (e.g. photoelectric and Compton effect) [3] or materials bases [4]. There are several ways to perform material decomposition. Threedimensional (3D) material density volumes can be retrieved from energy-resolved volumes, i.e., after the tomographic reconstruction [5]. Material decomposition can also be done in a one-step approach, i.e., simultaneously to the tomographic reconstruction [6], or in the projection domain, i.e., prior to the tomographic reconstruction. While most methods have been tested on simulations, the results provided on practical systems remains limited in terms of accuracy and signal to noise ratio due to the limited photon counts in each energy bin and the non linearity of the detector response [1, 4].

In this work, material decomposition in the projection domain is considered since it allows to decompose each projection in parallel. As in [7], we propose to address material decomposition as a nonlinear, ill-posed inverse problem. In [8], we proposed a Gauss-Newton algorithm to minimize a regularized cost function [8]. This approach enjoys a fast convergence but, contrary to [7], it does not ensure the solutions to be positive. Our contribution here is to provide a fast constrained algorithm.

More specifically, we investigate box constraints for which bounds are set from prior knowledge about the projected mass density of the different materials of the sample. Simple strategies for constrained optimization are available for first-order algorithms [9]. However, they usually suffer from slow convergence. In this paper, we introduce two versions of a fast second-order algorithm, referred to as projected Gauss-Newton with fixed bounds (PGN-FB) and with evolving bounds (PGN-EB). These algorithms are evaluated considering the projections of two realistic numerical phantoms and compared to a standard Gauss-Newton (GN) algorithm.

\section{MATERIAL DECOMPOSITION IN SPECTRAL CT}

\subsection{Forward model}

The following spectral CT forward model $[8,10]$ is considered:

$$
\mathbf{s}_{i}(\mathbf{u})=\int_{\mathbf{R}} d_{i}(E) n^{0}(E) \exp \left(-\sum_{m=1}^{M} a_{m}(\mathbf{u}) \tau_{m}(E)\right) \mathrm{d} E,
$$

where

$$
\mathbf{a}_{m}=\int_{\mathcal{L}(\mathbf{u})} \rho_{m}(\mathbf{x}) \mathrm{d} \mathbf{x}
$$

is the projection of all the mass of the material $m$ along the $\mathrm{X}$-ray path $\mathcal{L}(\mathbf{u}), \mathbf{s}_{i}(\mathbf{u})$ is the number of photons hitting the detector in the $i$-th energy bin at the pixel $\mathbf{u}, n^{0}(E)$ is the number of photons send to each energy $E, d_{i}(E)$ is the response function for the $i$-th energy bin, $\rho_{m}(\mathbf{x})$ is the mass of the material $m$ at the voxel $\mathbf{x}$ in $\mathrm{g} . \mathrm{cm}^{-3}$ and $\tau_{m}(E)$ is a well- 
chosen function that describes the attenuation of the material $m$ at energy $E$.

\subsection{Discretization and noise}

Assuming that the detector contains $P$ pixels and $I$ energy bins, the measured data can be gathered in a vector $\mathbf{s} \in \mathbf{R}^{I P}$

$$
\mathbf{s}=\left[s_{1,1}, \ldots, s_{I, 1}, \ldots, s_{I, P}\right]^{T}
$$

Similarly, the map of materials $\mathbf{a} \in \mathbf{R}^{M P}$ is defined by

$$
\mathbf{a}=\left[a_{1,1}, \ldots, a_{M, 1}, \ldots, a_{M, P}\right]^{T}
$$

\subsection{Inverse problem}

Material decomposition consists in recovering the material map a from the data $\mathbf{s}$. Let $\mathbf{s}=\mathcal{F}(\mathbf{a})$ be the forward model defined in Eq. (1). The map of materials is recovered minimizing the following cost function:

$$
\mathcal{C}(\mathbf{a})=\mathcal{D}(\mathbf{a})+\alpha \mathcal{R}(\mathbf{a})
$$

We consider a weighted least squares data fidelity term, i.e.,

$$
\mathcal{D}(\mathbf{a})=\frac{1}{2}\|\mathbf{W}(\mathbf{s}-\mathcal{F}(\mathbf{a}))\|^{2},
$$

where $\mathbf{W}=\operatorname{diag}\left(\frac{1}{\sqrt{\mathbf{s}}}\right)$ is the weighting matrix, and $\operatorname{diag}(\mathbf{x})$ is a diagonal matrix composed of the element of $\mathbf{x}$. The regularization term allows having a stabilized solution in the presence of the noise. Following [8] and considering three materials (soft tissues, bones and gadolinium as a marker), we choose

$$
\mathcal{R}(\mathbf{a})=\left\|\Delta \mathbf{a}_{\text {soft }}\right\|_{2}^{2}+\left\|\nabla \mathbf{a}_{\text {bone }}\right\|_{2}^{2}+\left\|\nabla \mathbf{a}_{\mathrm{Gd}}\right\|_{1}
$$

where $\nabla$ and $\Delta$ are first- and second-order differential operator. The operators $\|.\|_{2}$ and $\|.\|_{1}$ are the $\ell_{2}$ - and $\ell_{1}$-norm, respectively.

The cost function (5) can be efficiently minimized using the Gauss-Newton algorithm implemented in the SPRAY Matlab toolbox $[8,11]$ (denoted RWLS-GN in the original paper). It has been used as a reference to benchmark our proposed algorithm.

\section{CONSTRAINED NEWTON ALGORITHM}

In this paper, we investigate the resolution of the boxconstrained decomposition problem given by

$$
\min _{\mathbf{a}} \mathcal{C}(\mathbf{a}) \quad \text { s.t. } L_{m} \leq a_{m, p} \leq U_{m}, \forall m, \forall p
$$

where $L_{m}$ and $U_{m}$ are lower and upper bounds for the $m$-th material map. In particular, we consider iterative projection methods

$$
\mathbf{a}^{k+1}=\mathcal{P}_{\Omega}\left(\mathbf{a}^{k}-\lambda^{k} \delta \mathbf{a}^{k}\right)
$$

where $\delta \mathbf{a}^{k}$ is a descent direction, $\lambda^{k}$ is a step length, and $P_{\Omega}$ is projector onto $\Omega$ used to enforce the constraints. Here, we have $\Omega_{m}=\left[L_{m}, U_{m}\right]^{P}$ and $\Omega=\left(\Omega_{m}\right)_{1 \leq m \leq M}$. Note that second-order methods are considered, i.e., $\delta \mathbf{a}^{k}$ is computed using an approximation of the Hessian of the cost function $\mathcal{C}$ (or its inverse). In the following, let $\mathbf{H}^{k}$ and $\mathbf{g}^{k}$ denote the Hessian and the gradient of $\mathcal{C}$ at $\mathbf{a}^{k}$, respectively.

\subsection{Projected quasi Newton}

Kim et al. have introduced a projected quasi-Newton algorithm with box-type constraints [12]. As in [13], the descent direction is computed after partitioning the variables into two groups, free and fixed. However, the fixed variables are chosen using both the gradient and second-order information. The step length is computed by backtracking, i.e., by finding the smaller non-negative integer $t_{k}$ that verifies the following inequality [13]:

$$
\mathcal{C}\left(\mathbf{a}^{k}\right)-\mathcal{C}\left(\mathcal{P}_{\Omega}\left[\mathbf{a}^{k}-\gamma \sigma^{t_{k}} \delta \mathbf{a}^{k}\right]\right) \geq \tau \gamma \sigma^{t_{k}}\left(\mathbf{g}^{k}\right)^{\top} \delta \mathbf{a}^{k}
$$

where $\gamma>0, \sigma$ and $\tau \in(0,1)$ are some scalars. The step length is then set to $\lambda^{k}=\gamma \sigma^{t_{k}}$.

\subsection{Projected Gauss-Newton}

In comparison to the projected quasi Newton algorithm, our proposed projected Gauss-Newton exploits the Hessian of $\mathcal{C}$, not an approximation of its inverse obtained using first-order information only. The set of fixed variables is obtained using the gradient (see index set $\mathcal{I}_{1}^{k}$ in Algorithm 1) and also solving a linear system of equation involving both the Hessian $\mathbf{H}^{k}$ and the active gradient $\tilde{\mathbf{g}}^{k}$ (see index set $\mathcal{I}_{2}^{k}$ in Algorithm 1). The descent direction is computed solving a second system of linear equations. The Hessian being sparse [8], its inverse can be computed efficiently using dedicated solvers. This algorithm is referred to as projected Gauss-Newton with fixed bounds (PGN-FB) and is detailed in Algorithm 1.

As show in Section 5, PGN-FB may fail to converge to acceptable solutions. Hence, we propose a simple variant, referred to as PGN with evolving bounds (PGN-EB), that incorporates the constraints in a progressive manner. The key idea is to build a sequence of constraint sets that tend towards the desired constraint set $\Omega$ [14]. We define $\Omega_{m, k}=\left[\ell_{m}^{k}, U_{m}\right]^{P}$, where $\ell_{m}^{k}$ is the evolving lower bound for the $m$-th material, which is chosen so as to satisfy $\ell_{m}^{k} \leq L_{m}, \forall k$ and $\Omega_{k}=\left(\Omega_{m, k}\right)_{1 \leq m \leq M}$. Then, the resulting sequence of constraint sets is such that $\Omega_{0} \supset \Omega_{1} \cdots \supset \Omega$. In practice, the strategy described in Algorithm 2 is adopted. Starting from $\ell_{m}^{0}$, the lower bound is chosen at each iteration as the minimum of $L_{m}$ and $\mathbf{a}_{m}^{k}$. If the evolving lower bound for a given material is not increasing, i.e., if $\ell_{m}^{k+1}=\ell_{m}^{k}$, then it is steered towards $L_{m}$ by a percentage $p_{\min }$. $\ell_{m}^{0}$ is chosen very low to perform the first iteration without boundaries, and if the value is low enough it doesn't change the behavior of the algorithm. Finally, if the stopping criteria are satisfied and $\ell_{m} \neq L_{m}$ for any given material, the lower bounds are set to $L_{m}$ and one more iteration is made. Note that an evolving upper bound can be defined in a similar way.

\section{NUMERICAL EXPERIMENTS}

In this work, the proposed algorithms are evaluated on two numerical phantoms: an anthropomorphic thorax $(240 \times$ $185 \times 83$ voxels $)[15]$ and mouse $(104 \times 190 \times 496$ voxels $)$ [16]. Both are made of $M=3$ materials, namely soft tissues, 


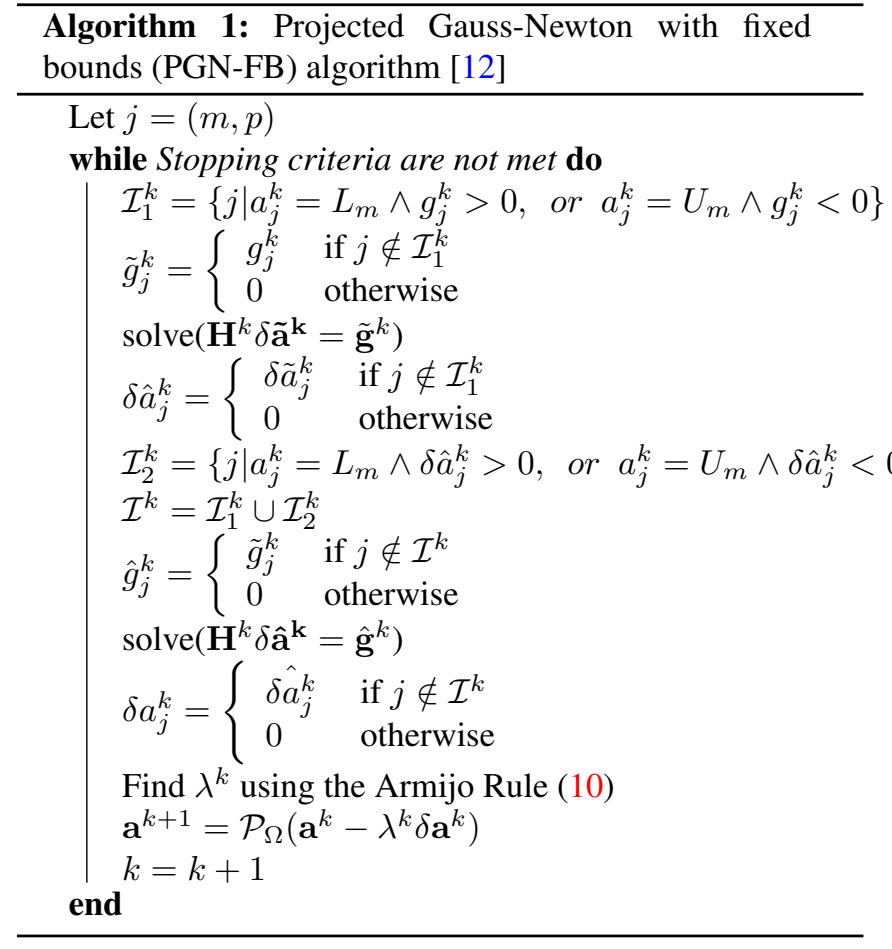

bones, and gadolinium. The gadolinium is placed into the portal vein of the thorax phantom and in the heart of the DigiMouse. Projections for the angles $\theta=\left\{0^{\circ}, 90^{\circ}\right\}$ are obtained using the radon Matlab's function.

We considered a $120-\mathrm{keV} \mathrm{X}$-ray source generated using the spekCalc software [17] and a photon counting detector with $I=4$ bins of energy. We define the number of photons sent at every pixel by $N^{0}=\int_{\mathbf{R}} n^{0}(E) \mathrm{d} E$. The DigiMouse phantom being less attenuating than the thorax phantom, different number of photons are considered. We choose $N^{0}=10^{7}$ photons for the thorax and $N^{0}=10^{4.5}$ photons for DigiMouse.

For both phantoms, all algorithms are initialized with the same uniform material maps $\left(\mathbf{a}_{\text {soft }}=1 \mathrm{~g} . \mathrm{cm}^{-2}\right.$ and $\mathbf{a}_{\text {bone }}=$ $\mathbf{a}_{\mathrm{Gd}}=0 \mathrm{~g} . \mathrm{cm}^{-2}$ ). All algorithms are stopped if the relative decrease of the cost function is lower than $0.1 \%$, or if $\lambda^{k}$ is lower than $5.10^{-3}$, or if the iteration number $k$ is larger than 150. Bounds are set to $L_{m}=0 \mathrm{~g} . \mathrm{cm}^{-2}$ and $U_{m}=50$ g.cm ${ }^{-2}$. For PGN-EB, $\ell_{m}^{0}=-50$ g.cm ${ }^{-2}$ and the percentage $p_{\text {min }}$ is set to $20 \%$.

Since the ground truth is known, a relative decomposition error $\xi_{\theta}$ of the $\theta$-th projection can be computed as:

$$
\xi_{\theta}=\sum_{m=1}^{M} \frac{\left\|\mathbf{a}_{m}^{\mathrm{dec}}(\theta)-\mathbf{a}_{m}^{\text {true }}(\theta)\right\|}{\left\|\mathbf{a}_{m}^{\text {true }}(\theta)\right\|}
$$

A wide range of regularization parameter were tested, $\alpha \in$ $\left[10^{-2}, 10^{2}\right]$. The choice of the optimal regularization parameter was performed following [18], i.e the regularization parameter was chosen such that $\mathcal{D}\left(\mathbf{a}_{\alpha}\right) \simeq P \times I$, where $P \times I$ represents the number of noisy Poisson variables.

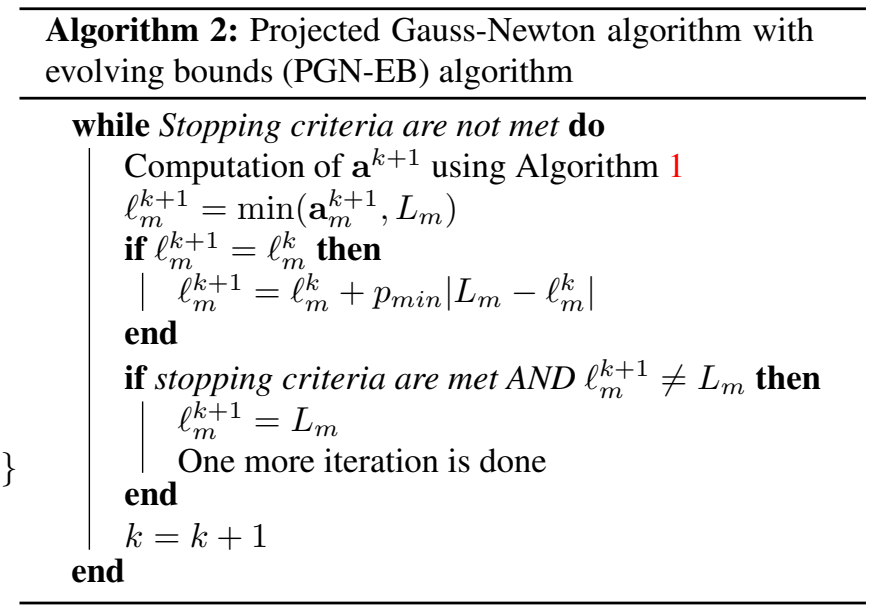

\begin{tabular}{|c|l|c|c|c|c|}
\cline { 3 - 6 } \multicolumn{2}{c|}{} & \multicolumn{2}{c|}{$\xi_{\theta}$} & \multicolumn{2}{c|}{ CPU Time (second) } \\
\hline$\theta$ & Algorithm & DigiMouse & Thorax & DigiMouse & Thorax \\
\hline \multirow{3}{*}{0} & GN & 1.08 & 1.66 & 23.5 & 23.5 \\
\cline { 2 - 6 } & PGN-EB & $\mathbf{0 . 6 2}$ & $\mathbf{1 . 2 1}$ & 55.7 & 15.8 \\
\cline { 2 - 6 } & PGN-FB & 116 & 4.35 & $\mathbf{6 . 4}$ & $\mathbf{3 . 9 3}$ \\
\hline \multirow{3}{*}{$90^{\circ}$} & GN & 1.18 & 1.41 & 22.6 & 21.5 \\
\cline { 2 - 6 } & PGN-EB & $\mathbf{0 . 7 3}$ & $\mathbf{1 . 0 1}$ & 51.5 & 10.8 \\
\cline { 2 - 6 } & PGN-FB & 84.02 & 3.25 & $\mathbf{5 . 7}$ & $\mathbf{5 . 5 2}$ \\
\hline
\end{tabular}

Table 1. Relative decomposition error for all algorithms and both phantoms, considering two different $\theta$.

\section{RESULTS AND DISCUSSION}

The relative decomposition errors $\xi_{\theta}$ and CPU time are reported in Table 1 , for both angles $\theta$ and both phantoms. It is observed that PGN-EB achieves the smallest error between the three algorithms, regardless the phantom and view angle. Moreover, PGN-FB leads to a very bad decomposition since it constraints the solution too much. We can see that PGNEB is slower than GN since it needs to solve two more linear equations than GN. The difference is noticeable for large dimension images, e.g. for DigiMouse.

The number of iterations for PGN-EB and GN are similar. For DigiMouse PGN-EB converges after 6 iterations and GN after 5. For the thorax phantom, the GN algorithm took 25 iterations to converge while PGN-EB needs only 9.

Figure 1 shows the decomposition of DigiMouse for $\theta=$ $0^{\circ}$ (left) and $\theta=90^{\circ}$ (right). There is a better contrast with PGN-EB than with GN since the background is more like what we can expect with PGN-EB. Similar results are obtained with the thorax phantom but are not shown here, due to limited space.

\section{CONCLUSION}

In this work, we introduce a fast algorithm for material decomposition in spectral CT. It is a simple modification of a standard Gauss-Newton (GN) algorithm, which can enforce box constraints iteratively. Compared to GN, it improves the three-material decomposition of a mouse and thorax phantoms. This could benefit to applications where only projec- 


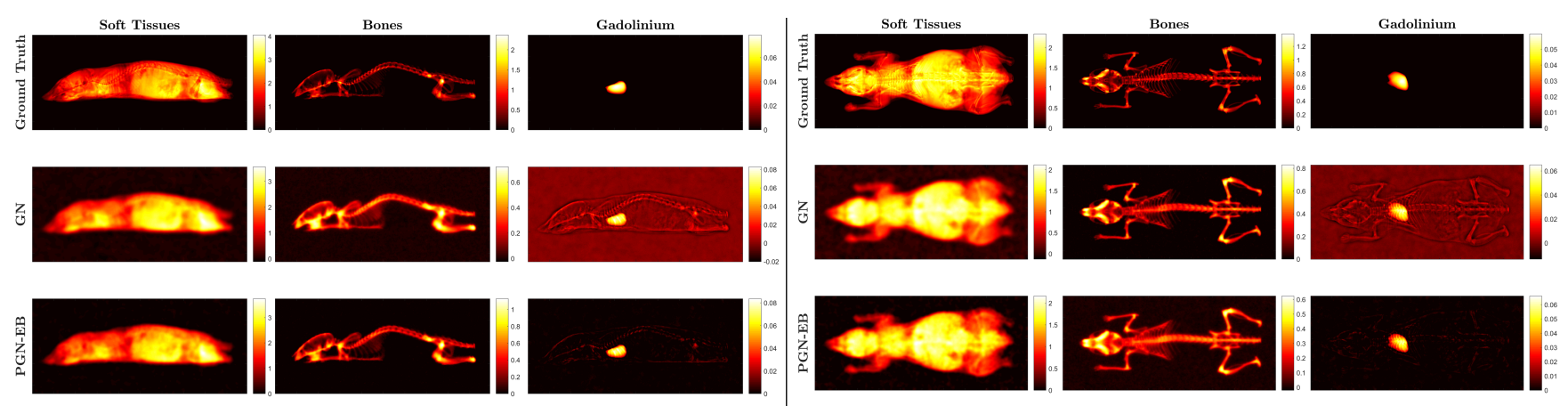

Fig. 1. Decomposition of DigiMouse $\left(\theta=0^{\circ}\right.$ (left) and $\theta=90^{\circ}$ (right)). Ground truth (top row), GN (middle row) and PGN-EB (bottom row) for the 3 different materials: soft tissues (left), bones (middle) and gadolinium (right), for each angle.

tions are considered (e.g. interventional radiology or airport security). In the future, we will address tomographic reconstructions from decomposed projections. We anticipate that improved projected maps result in improved density maps.

\section{ACKNOWLEDGMENT}

The authors would like to thank the anonymous reviewers for their careful reading. This work was supported by the LABEX PRIMES (ANR-11-LABX-0063) of Universite de Lyon, within the program "Investissements d'Avenir" (ANR-11-IDEX-0007) operated by the French National Research Agency (ANR).

\section{REFERENCES}

[1] K. Taguchi and Jan S. Iwanczyk, "Vision 20/20: Single photon counting x-ray detectors in medical imaging," Med. Phys, vol. 40, no. 10, 2013.

[2] D P Cormode, E Roessl, A Thran, T Skajaa, R E Gordon, JP Schlomka, V Fuster, E A Fisher, W JM Mulder, R Proksa, et al., "Atherosclerotic plaque composition: analysis with multicolor ct and targeted gold nanoparticles 1," Radiology, vol. 256, no. 3, pp. 774-782, 2010.

[3] R.E. Alvarez and A. Macovski, "Energy-selective reconstructions in x-ray computerised tomography," $P M B$, vol. 21, no. 5, pp. 733, 1976.

[4] J. Schlomka, E. Roessl, R. Dorscheid, S. Dill, G. Martens, T. Istel, C. Bäumer, C. Herrmann, R. Steadman, G. Zeitler, et al., "Experimental feasibility of multi-energy photoncounting k-edge imaging in pre-clinical computed tomography," $P M B$, vol. 53, no. 15, pp. 4031, 2008.

[5] C. Maaß, M. Baer, and M. Kachelrieß, "Image-based dual energy ct using optimized precorrection functions: A practical new approach of material decomposition in image domain," Med. Phys, vol. 36, no. 8, pp. 3818-3829, 2009.

[6] Y. Long and J.A. Fessler, "Multi-material decomposition using statistical image reconstruction for spectral ct," IEEE TMI, vol. 33, no. 8, pp. 1614-1626, 2014.

[7] J. Noh, J.A. Fessler, and P.E. Kinahan, "Statistical sinogram restoration in dual-energy ct for pet attenuation correction," IEEE transactions on medical imaging, vol. 28, no. 11, pp. 1688-1702, 2009.
[8] N. Ducros, J.F.P.J. Abascal, B. Sixou, S. Rit, and F. Peyrin, "Regularization of nonlinear decomposition of spectral x-ray projection images," Med. Phys, vol. 44, no. 9, 2017.

[9] J.B. Rosen, "The gradient projection method for nonlinear programming. part i. linear constraints," JSIAM, vol. 8, no. 1, pp. 181-217, 1960.

[10] E. Roessl, B. Brendel, K-J. Engel, J-P. Schlomka, A. Thran, and R. Proksa, "Sensitivity of photon-counting based-edge imaging in x-ray computed tomography," IEEE TMI, vol. 30, no. 9, pp. 1678-1690, 2011.

[11] "Spray toolbox," https://www.creatis.insa-lyon.fr/ ucros/WebPage/spray.html.

[12] D. Kim, S. Sra, and I.S. Dhillon, "Tackling box-constrained optimization via a new projected quasi-newton approach," SIAM Journal on Scientific Computing, vol. 32, no. 6, pp. 3548-3563, 2010.

[13] D.P. Bertsekas, Nonlinear programming, Athena scientific Belmont, 1999.

[14] A.B. Levy, "Constraint incorporation in optimization," Mathematical Programming, vol. 110, no. 3, pp. 615-639, 2007.

[15] R. Kéchichian, S. Valette, M. Desvignes, and R. Prost, "Shortest-path constraints for $3 \mathrm{~d}$ multiobject semiautomatic segmentation via clustering and graph cut," IEEE TIP, vol. 22, no. 11, pp. 4224-4236, 2013.

[16] B. Dogdas, D. Stout, A.F. Chatziioannou, and R.M. Leahy, "Digimouse: a 3d whole body mouse atlas from CT and cryosection data," $P M B$, vol. 52, no. 3, pp. 577, 2007.

[17] G Poludniowski, G Landry, F DeBlois, PM Evans, and F Verhaegen, "Spekcalc: a program to calculate photon spectra from tungsten anode x-ray tubes," $P M B$, vol. 54, no. 19, 2009.

[18] J.M Bardsley and J. Goldes, "Regularization parameter selection methods for ill-posed poisson maximum likelihood estimation,” Inverse Problems, vol. 25, no. 9, pp. 095005, 2009. 